\title{
Numerical Evaluation of the Green's functions for Arbitrarily Shaped Enclosures
}

\author{
J.S. Gómez-Díaz, M. Martínez-Mendoza, F.D. Quesada-Pereira, J. Pascual-Garcia, \\ F. J. Perez-Soler and A. Alvarez-Melcon \\ Technical University of Cartagena, Antiguo Cuartel de Antigones \\ 30202 Cartagena, Murcia, Spain. \\ E-mail: madhap@ono.com
}

\begin{abstract}
In this paper, a new spatial method has been implemented for the efficient calculation of the mixed potential Green's functions associated to electrical sources, when they are placed inside arbitrarily-shaped cylindrical cavities. The technique consists of placing electric dipole images and charges outside the cavity, imposing, at discrete points of the metallic wall, the appropriate boundary conditions for the potentials. Results show that the numerical convergence is attained fast. The cut-off frequencies and potential patterns for a trapezium-shaped waveguide are compared to those obtained by a standard finite elements technique, showing excellent agreement. Furthermore, a printed planar filter shielded in a square cavity has been analyzed with the new Green's functions, showing the practical value of the new theory.
\end{abstract}

Index Terms-Green functions, Cavities, Waveguides, Waveguide filters, Printed Circuits

\section{INTRODUCTION}

The interest in studying techniques which allow for the prediction of the electrical behavior of shielded printed circuits and cavity backed antennas has increased in the last years. This is because of the increasing popularity of these devices for use in practical communication systems [1]. For the analysis of these components, the integral equation technique has grown in popularity due to its efficiency, and to the capability to push to a maximum the analytical features of the problem [2].

The formulation of the integral equation requires an algorithm which leads to the computation of the electromagnetic scalar and vector potentials of the problem, under point source excitations (i.e. the so called mixed potentials Green's functions). For the calculation of the Green's functions, only the rectangular enclosure has been extensively treated in the past ([3]). One possibility is to express the Green's functions in terms of spectral domain slowly convergent series of vector modal functions ([4]). However, spatial domain formulations can also be used to compute the Green's functions, expressing them as slow convergent series of spatial images ([5]).

On the other hand, a new spatial domain method for the Green's function computation inside circular cylindrical cavities was recently proposed in [6] and in [7]. The technique uses image theory to enforce the proper boundary conditions for the fields. However, the Green's functions formulation inside arbitrarily shaped cavities has never been treated in the past, and one has to resort to pure numerical techniques, such as finite elements or finite differences, to treat this kind of problems.

In this context, an extension of the original image theory proposed in [6] is presented in this paper. This new technique permits the calculation of the mixed potential Green's functions in arbitrarily-shaped geometries. In this contribution, the new formulation to compute both the electric scalar potential and the magnetic vector potential dyadic Green's functions produced by electric currents inside arbitrarily shaped cavities is shown. Resonant frequencies can also be computed with this new technique, and some useful results are given. In addition, a comparison between the potentials obtained with the novel image technique and the electric field computed with a finite elements technique, inside a trapezium-shaped cavity, is presented. Finally, a microstrip filter shielded in a square cavity has been efficiently analyzed, showing the accuracy and practical value of the technique proposed.

\section{THEORY}

A trapezium-shaped cylindrical metallic cavity is depicted in Fig. 1 in order to introduce the spatial images mixed potential Green's functions formulation, without lack of generality.

For the electric scalar potential we should impose null potential on the cavity wall. If the boundary condition is imposed at only one point, then only one charge image will be used; this situation is shown in Fig. 1. The idea is to impose the boundary conditions at $N$ points on the cavity wall, using $N$ charge images placed outside the cavity. The key point of the procedure is to evaluate numerically the value of the $N$ charges so that the boundary conditions for the electric scalar potential are satisfied at the $N$ tangent points. The following system of linear equations is obtained:

$$
\sum_{k=1}^{N} q_{k} G_{V}\left(\vec{r}_{i}, \vec{r}_{k}{ }^{\prime}\right)=-G_{V}\left(\vec{r}_{i}, \vec{r}_{0}{ }^{\prime}\right) ; \quad i=1,2,3, \ldots, N
$$




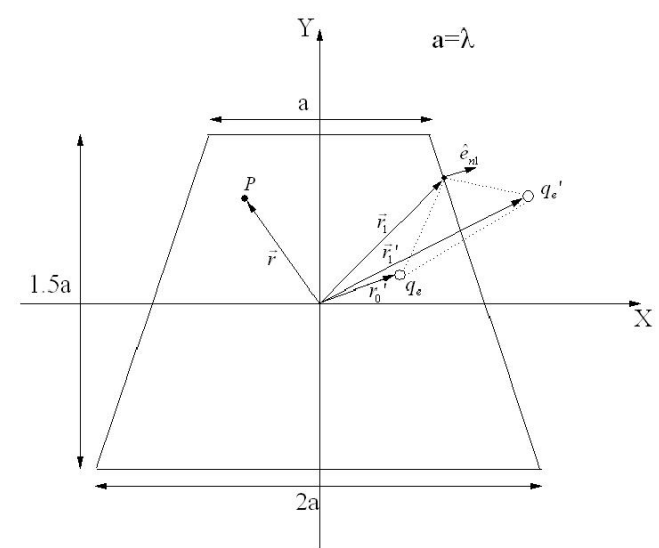

Fig. 1. Image charge arrangement used to enforce the boundary conditions for the electric scalar potential along the cylindrical wall. Point $P$ is a generic observation point

where all position vectors are shown in Fig. 1, and $G_{V}\left(\vec{r}, \vec{r}^{\prime}\right)$ is the potential Green's function of a unit point charge in free-space. Real cavities, including dielectric layers, can also be studied using the multilayered media spatial domain Green's functions, obtained through Sommerfeld integrals [8].

The solution of this system provides the complex values of the $N$-images charges $\left(q_{k}\right)$ needed to satisfy the potential boundary conditions at $N$-different points on the wall. The final electric scalar potential Green's function inside the cavity is evaluated by reusing the already computed charge amplitudes:

$$
G_{V_{c y}}(\vec{r})=G_{V}\left(\vec{r}, \vec{r}_{0}{ }^{\prime}\right)+\sum_{k=1}^{N} q_{k} G_{V}\left(\vec{r}, \vec{r}_{k}{ }^{\prime}\right)
$$

For the evaluation of the magnetic vector potential dyadic Green's function, a similar procedure is followed. We recall that the final boundary condition to impose is the zero tangent component of the electric field on the cavity wall. This condition leads to two different conditions for the magnetic vector potential:

$$
\hat{e}_{n} \times \vec{A}=0 ; \quad \nabla \cdot \vec{A}=0
$$

Where $\hat{e}_{n}$ is the unit vector normal to the wall (see Fig. 2). If the first equation is satisfied, the second is simplified, working in rectangular coordinates, to a single condition involving the magnetic vector potential normal component, as follows:

$$
(\nabla \cdot \vec{A}) \cdot \hat{e}_{n}=0
$$

Considering a unit dipole oriented along the $x$-axis, the utilization of two orthogonally oriented electric dipole images is proposed in order to impose both conditions at one point of the cylindrical wall (see Fig. 2). This procedure can now be generalized, to impose the right boundary conditions at $N$-points along the cavity wall. In

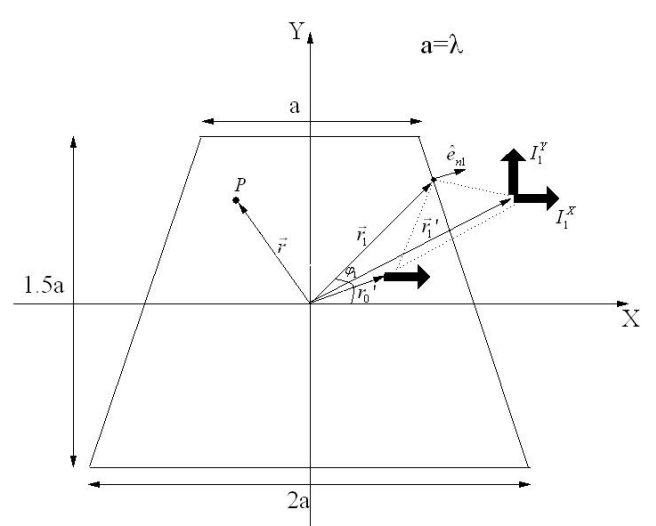

Fig. 2. Electric images dipoles used to enforce the boundary conditions for the mannetic vector potential at a discrete point on the trapeziumshaped cavity wall.

this way, $N$-image dipoles are used, obtaining a $2 N \times 2 N$ system of linear equations:

$$
\begin{gathered}
-\sin \varphi_{i} \sum_{k=1}^{N} G_{A}^{x x}\left(\vec{r}_{i}, \vec{r}_{k}{ }^{\prime}\right) I_{k}^{x}+\cos \varphi_{i} \sum_{k=1}^{N} G_{A}^{y y}\left(\vec{r}_{i}, \vec{r}_{k}{ }^{\prime}\right) I_{k}^{y}= \\
\sin \varphi_{i} G_{A}^{x x}\left(\vec{r}_{i}, \vec{r}_{0}^{\prime}\right) \\
\cos \varphi_{i} \sum_{k=1}^{N} C_{i, k}^{x} I_{k}^{x}+\sin \varphi_{i} \sum_{k=1}^{N} C_{i, k}^{y} I_{k}^{y}= \\
-\cos \varphi_{i} C_{i, 0}^{y} ; \quad i=1,2,3, \ldots, N
\end{gathered}
$$

where the following constants have been defined:

$$
\begin{aligned}
& C_{i, k}^{x}=\cos \varphi_{i} \frac{\partial G_{A}^{x x}\left(\vec{r}_{i}, \vec{r}_{k}{ }^{\prime}\right)}{\partial x}+\sin \varphi_{i} \frac{\partial G_{A}^{x x}\left(\vec{r}_{i}, \vec{r}_{k}{ }^{\prime}\right)}{\partial y} \\
& C_{i, k}^{y}=\sin \varphi_{i} \frac{\partial G_{A}^{y y}\left(\vec{r}_{i}, \vec{r}_{k}{ }^{\prime}\right)}{\partial y}+\sin \varphi_{i} \frac{\partial G_{A}^{y y}\left(\vec{r}_{i}, \vec{r}_{k}{ }^{\prime}\right)}{\partial x}
\end{aligned}
$$

Both constants can be computed, for a general multilayered medium, using the spectral domain formulation combined with the Sommerfeld integral transformation. For the free space case, they can be calculated in closed form as:

$$
\begin{aligned}
& \frac{\partial G_{A}^{x x}\left(\vec{r}_{i}, \vec{r}_{k}{ }^{\prime}\right)}{\partial x}=\frac{\partial G_{A}^{y y}\left(\vec{r}_{i}, \vec{r}_{k}{ }^{\prime}\right)}{\partial x}= \\
& -\frac{\mu_{0}}{4 \pi} \frac{e^{-j k_{0}\left|\vec{r}_{i}-\vec{r}_{k}{ }^{\prime}\right|}\left(x_{i}-x_{k}^{\prime}\right)\left(1+j k_{0}\left|\vec{r}_{i}-\vec{r}_{k}{ }^{\prime}\right|\right)}{\left|\vec{r}_{i}-\vec{r}_{k}\right|^{3}} \\
& \frac{\partial G_{A}^{y y}\left(\vec{r}_{i}, \vec{r}_{k}{ }^{\prime}\right)}{\partial y}=\frac{\partial G_{A}^{x x}\left(\vec{r}_{i}, \vec{r}_{k}{ }^{\prime}\right)}{\partial y}= \\
& -\frac{\mu_{0}}{4 \pi} \frac{e^{-j k_{0}\left|\vec{r}_{i}-\vec{r}_{k}{ }^{\prime}\right|}\left(y_{i}-y_{k}^{\prime}\right)\left(1+j k_{0}\left|\vec{r}_{i}-\vec{r}_{k}{ }^{\prime}\right|\right)}{\left|\vec{r}_{i}-\vec{r}_{k}{ }^{\prime}\right|^{3}}
\end{aligned}
$$

Once the system is solved, the magnetic vector potential is recovered inside the arbitrary geometry by using all the 
amplitudes of the $(2 N)$ image electric dipoles $\left(I_{k}^{x}, I_{k}^{y}\right)$ :

$$
\begin{aligned}
& G_{A_{c y}}^{x x}\left(\vec{r}, \vec{r}_{0}{ }^{\prime}\right)=G_{A}^{x x}\left(\vec{r}, \vec{r}_{0}{ }^{\prime}\right)+\sum_{k=1}^{N} I_{k}^{x} G_{A}^{x x}\left(\vec{r}_{i}, \vec{r}_{k}{ }^{\prime}\right) \\
& G_{A_{c y}}^{y x}\left(\vec{r}, \vec{r}_{0}{ }^{\prime}\right)=\sum_{k=1}^{N} I_{k}^{y} G_{A}^{y y}\left(\vec{r}_{i}, \vec{r}_{k}{ }^{\prime}\right)
\end{aligned}
$$

It is worth mentioning that all the equations are independent on the geometry of the waveguide, because a specific coordinates system is not used, and a fixed location of the images and the tangent points is not assumed. The selection of the tangent points and the distribution of the images surrounding the geometry is also important for the convergence of the algorithm. Since the proposed formulation pretends to be useful for arbitrarily geometries, the situation of the images will vary depending on the waveguide shape.

As already said, the spatial images technique can easily be extended to consider a multilayered medium. This can be done by replacing the free-space Green's functions with the multilayered media Green's functions formulated in the spatial domain through the well known Sommerfeld tranformation [8]. This allows to impose automatically the boundary conditions at the top and the bottom covers, and to take into account for the presence of dielectric layers [6].

\section{RESULTS}

In order to analyze the trapezium-shaped cavity presented in Fig. 1 as a real three-dimensional enclosure, the cavity height (z-axis in Fig. 3 ) is taken as $0.4 \lambda$. In this case, 31 images are used to enforce the boundary conditions on the wall of the trapezium-shaped cavity, as shown in Fig. 3. To obtain the resonances of the cavity,

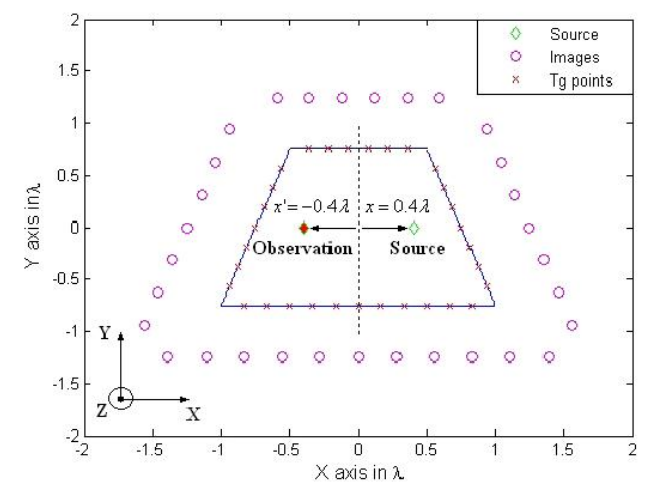

Fig. 3. Trapezium waveguide analyzed with 31 images. Source is placed at point $(0.4 \lambda, 0)$ and Observation is at point $(-0.4 \lambda, 0)$.

the potentials are represented as a function of frequency for a fixed position of source and observation points (see Fig. 3). Resonant frequencies can be clearly observed in Fig. 4, corresponding to sharp peaks in the response.

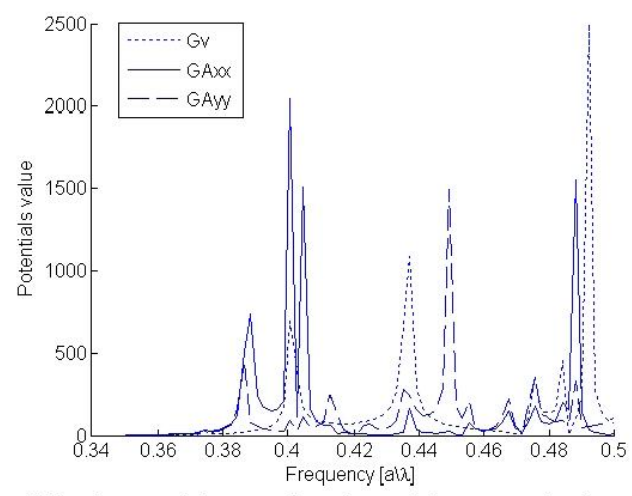

Fig. 4. Mixed potentials as a function of frequency in the trapeziumshaped cavity shown in Fig. 3.

TABLE I

CUT-OFF FREQUENCIES FOR CAVITY IN FIG. 4.

\begin{tabular}{|c|c|c|}
\hline $\begin{array}{c}\text { Cut-off frequencies, } \\
\text { HFSS } \odot\left(\frac{a}{\lambda}\right)\end{array}$ & $\begin{array}{c}\text { Cut-off frequencies, } \\
\text { Spatial images }\left(\frac{a}{\lambda}\right)\end{array}$ & $\begin{array}{c}\text { Relative } \\
\text { Difference }(\%)\end{array}$ \\
\hline \hline 0.3878 & 0.3885 & 0.1805 \\
\hline 0.4012 & 0.4007 & 0.1246 \\
\hline 0.4049 & 0.4047 & 0.0494 \\
\hline 0.4376 & 0.4372 & 0.0914 \\
\hline 0.4499 & 0.4493 & 0.1333 \\
\hline 0.4757 & 0.4747 & 0.2102 \\
\hline 0.4836 & 0.4831 & 0.1033 \\
\hline 0.4870 & 0.4878 & 0.1642 \\
\hline 0.4910 & 0.4919 & 0.1832 \\
\hline
\end{tabular}

The resonant frequencies predicted with the novel space image technique are compared to those obtained by a finite elements technique in Table I. High accuracy has been achieved, maintaining in all cases a relative error below $0.21 \%$.

To show that this technique can compute the potentials (even at the cavity resonances) without any converge problems, the electric scalar potential inside the trapeziumshaped cavity is depicted at the middle-height plane, and at the normalized frequency of: $\frac{a}{\lambda}=0.4831$. The results in Fig. 5 are compared with those obtained by a

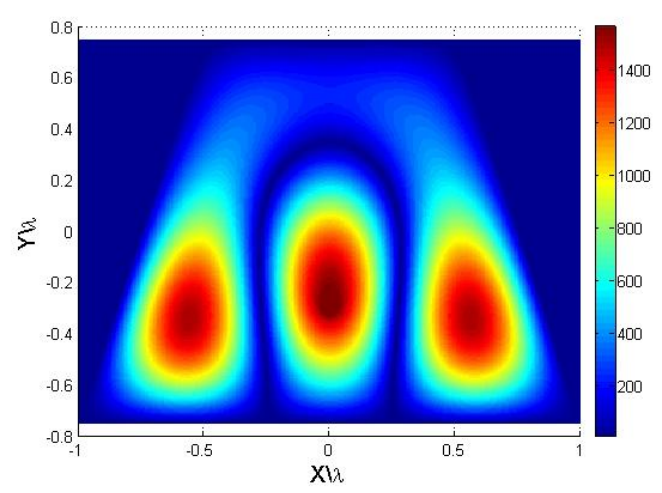

Fig. 5. Electric scalar potential obtained with the new spatial images technique at the normalized resonant frequency $\frac{a}{\lambda}=0.4831$. 
commercial finite elements HFSS ${ }^{(\mathcal{C}}$ method in Fig. 6. The same distribution of the $T M_{311}$ mode is observed with both methods.

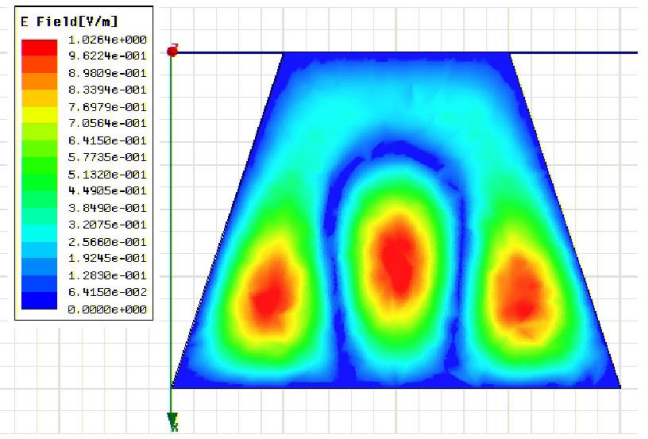

Fig. 6. Electric field z-component obtained by HFSS $\left.{ }^{(}\right)$at the same normalized frequency of $\frac{a}{\lambda}=0.4831$.

The technique developed can also be used for the analysis of practical printed circuits shielded in arbitrarilyshaped enclosures. A shielded band-pass filter based on coupled lines sections inside a square box is presented in Fig. 7. For this simple geometry, only 12 images are

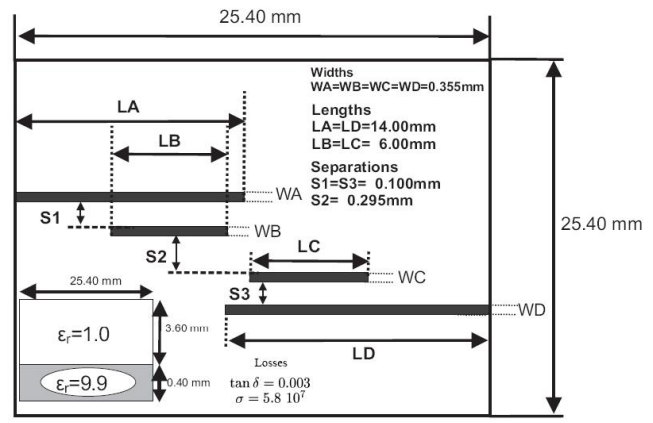

Fig. 7. Boxed microstrip band-pass filter based on coupled lines sections.

needed to obtain good convergence. The images are placed at the dielectric height, surrounding the structure. Scattering parameters are depicted in Fig. 8, showing an excellent agreement between our results and those obtained with a spectral domain technique presented in [9].

\section{CONCLUSIONS}

In this paper, we have presented a novel technique for the evaluation of the boxed Green's function in arbitrarilyshaped cylindrical cavities. The approach is based on the imposition of the boundary conditions for the mixed potential Green's functions at discrete points of the cavity wall. This imposition is achieved with the aid of discrete spatial images. The technique has been formulated entirely in the spatial domain and it has shown to converge rapidly. For validation, cut-off frequencies and potential patterns from a trapezium waveguide are compared to those obtained by

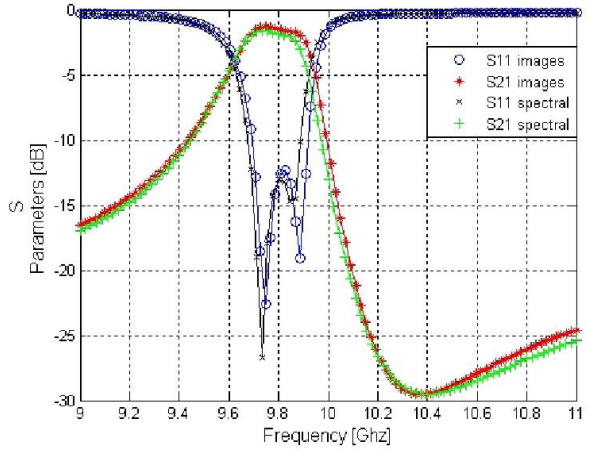

Fig. 8. Results for the band-pass filter shown in Fig, 7. Results from a spectral method are included as a validation

$\mathrm{HFSS}^{\complement}{ }^{\circledR}$. Moreover, a practical microwave bandpass filter inside a square cavity is analyzed, confirming the accuracy of the method, and its usefulness when modeling practical circuits.

\section{ACKNOWLEDGMENT}

This work has been supported by Spanish National project CICYT-TEC2004-04313-C02-02/TCM, and by Fundacion Seneca project, Ref. 02972/PI/05

\section{REFERENCES}

[1] R.-J. Mao and X.-H. Tang, "Novel dual-mode bandpass filters using hexagonal loop resonators," IEEE Transactions on Microwave Theory and Techniques, vol. 54, pp. 3526-3533, September 2006.

[2] G. G. Gentili, L. E. Garcia-Castillo, M. Salazar-Palma, and F. PerezMartinez, "Green's funcion analysis of single and stacked rectangular microstrip patch antennas enclosed in a cavity," IEEE Transactions on Antennas and Propagation, vol. 45, pp. 573-579, April 1997.

[3] G. V. Eleftheriades and J. R. Mosig, "On the network characterization of planar passive circuits using the method of moments," IEEE Transactions on Microwave Theory and Techniques, vol, 44, pp. 438445, March 1996.

[4] L. P. Dunleavy and P. B. Katehi, "A generalized method for analyzing shielded thin microstrip discontinuities," IEEE Transactions on Microwave Theory and Techniques, vol. 36, pp. 1758-1766, December 1988.

[5] A. A. Melcon and J. R. Mosig, "A novel spatial images technique for the analysis of cavity backed antennas," ACES, Applied Computational Electromagnetics Society, vol, 14, March 1999.

[6] F. Q. Pereira, P. V. Castejon, D. C. Rebenaque, J. P. Garcia, and A. A. Melcon, "Numerical evaluation of the Green's functions for cylindrical enclosures," IEEE Transactions on Microwave Theory and Techniques, vol. 53, pp. 94-105, January 2005.

[7] P. V. Castejon, F. Q. Pereira, D. C. Rebenaque, J. P. Garcia, and A. A. Melcon, "Numerical evaluation of the Green's functions for cylindrical enclosures by a new spatial images method," in IEEE, International Microwave Symposium, (Fort Worth, Texas, USA), 611 June 2004. No. TH5A-6.

[8] K. A. Michalski and J. R. Mosig, "Multilayered media Green's functions in integral equation formulations," IEEE Transactions on Antennas and Propagation, vol. 45, pp. 508-519, March 1997.

[9] A. A. Melcon, J. R. Mosig, and M. Guglielmi, "Efficient CAD of boxed microwave circuits based on arbitrary rectangular elements," IEEE Transactions on Microwave Theory and Techniques, vol. 47, pp. 1045-1058, July 1999. 